\title{
SURFACE MORPHOLOGY EVOLUTION IN STRESSED SOLIDS : SURFACE DIFFUSION CONTROLLED CRACK INITIATION
}

\author{
W. H. YANG $\dagger$ and D. J. SROLOVITZ †† \\ $\dagger$ Department of Material Science and Engineering, The University of Michigan, Ann Arbor, \\ MI 48019-2136, U.S.A. \\ $\ddagger$ Department of Materials and Interfaces, Weizmann Institute of Science, Rehovot 76100, Israel
}

(Received 28 December 1993; in revised form 21 May 1994)

\begin{abstract}
We present a nonlinear analysis of the temporal evolution of the surface morphology of a stressed solid based on a general parametric description of the surface shape. We find that surfaces of elastic, defect-free solids are unstable against the nucleation and growth of cracks. The rate at which this surface instability occurs depends on the material transport kinetic mechanism. The surface instability creates a groove that sharpens as it grows deeper. The groove growth rate accelerates until the groove reaches a critical length (or time) where the growth rate diverges. Comparison of these results with predictions of linear elastic fracture mechanics shows that the critical length is in excellent agreement with the classical Griffith fracture criterion, with no adjustable parameters. The stress field ahead of the growing groove becomes increasingly singular as the groove grows. Once the critical groove length is achieved, the stress field ahead of the groove approaches the inverse square root dependence on distance from the tip, which is characteristic of a sharp crack. Therefore, the sub-critical groove is not simply a short crack.
\end{abstract}

\section{INTRODUCTION}

Although a macroscopically flat surface bounding a semi-infinite solid is stable against small amplitude shape perturbations when the surface tension is finite (Mullins, 1959), the surface of a stressed solid is unstable against shape perturbations of sufficiently large wavelength (Asaro and Tiller, 1972; Grinfeld, 1986; Srolovitz, 1989). Such instabilities result from the competition between surface tension and elastic energy. The rate at which these unstable surface perturbations form and grow is controlled by the kinetics of material transport, such as by surface diffusion, bulk diffusion, evaporation, dissolution, etc.

The study of stress-driven morphological instabilities in solids dates back to the work of Hillig and Charles (1965) on stress corrosion cracking. They considered the case of an elliptical cavity in a remotely loaded solid body, where material transport was controlled by a surface chemical dissolution process. Rice and Chuang (1981) examined the variation of the chemical potential at a void and the energy-release rates associated with diffusive cavity growth and the subsequent formation of creep cracks from voids. In both cases, surface diffusion and/or chemical reactions have been shown to transform voids or notches into sharp cracks, when the stress is sufficiently 
large. If the stress is too small, however, the voids or notches become increasingly blunt. Heidug (1991) presented a thermodynamic analysis of the equilibrium and stability of solid-fluid phase boundaries under both stress and capillary effects. He demonstrated that curved interfaces in stressed solids are either metastable or unstable.

Asaro and Tiller (1972), Grinfeld (1986), and Srolovitz (1989) performed linear stability analyses for a surface bounding a two-dimensional semi-infinite solid under stress-assisted surface diffusion. They found that the competition between elastic strain energy and surface energy can lead to the growth of surface profile perturbations at large stresses, small surface tensions and/or long wavelengths. Gao (1991) has considered the stabilizing effects of gravity on the instability. Grilhe (1993) used a variational analysis to show that the symmetry between tensile and compressive stress for the onset of the surface instability is broken by the presence of surface tension. Spencer et al. (1991) and Freund and Jonsdottir (1993) have extended these stability analyses to include the case of an epitaxially strained solid film on a substrate.

Experimental investigations have corroborated several of the theoretical predictions regarding this strain energy driven surface instability. Torii and Balibar (1992) performed a series of experiments on nonhydrostatically stressed ${ }^{4} \mathrm{He}$ crystals and found that above a threshold stress, large grooves form on the surface of the crystal. A similar observation was made by Berrehar et al. (1992) who showed that a uniaxially stressed, epitaxial single crystal film of polymerized polydiacetylene on a monomer substrate forms regular grooves or quasi-periodic "cracks". Korsukov et al. (1992) studied the formation and growth of roughness on the surface of a stressed amorphous iron alloy. They found that following the removal of the applied stress, the surface roughness decayed away. Recently, Volkert (1993) showed that ion implanted, compressively stressed $\mathrm{SiO}_{2}$ is susceptible to forming this same type of crack-like surface morphology. Since these recent observations of a crack-like surface morphology were made in a wide variety of materials, it is clear that the development of this type of surface morphology does not depend on the detailed properties of the material or the operative matter transport mechanism.

These recent experiments further demonstrate that this stress driven instability leads to the formation of sharp, crack-like surface features. While the linear stability analyses do adequately predict the conditions for the onset of the surface instability, they are incapable of addressing the important issue of what type of surface morphology forms. A description of the late-time surface evolution requires inclusion of the important nonlinearities in the surface morphology evolution equations which are ignored in the linear stability analyses. Spencer et al. (1993) have studied the surface evolution of a stressed film on a rigid substrate based on a long-wave, nonlinear theory but did not observe the formation of sharp, crack-like features. Yang and Srolovitz (1993) applied a general numerical procedure to the full nonlinear surface evolution equations to examine the surface instability in a two-dimensional semi-infinite stressed solid. They showed that the stress-driven surface diffusion-controlled surface instability develops into a deep, crack-like groove morphology. Spencer and Meiron (1993) recently confirmed this result using another numerical method. Assuming that the surface profile may be described as a cycloid, Chin and Gao (1993) have shown that the energy of cycloid surface morphologies decreases as the cycloid sharpens. 
In this paper, we present the results of a numerical study of the full nonlinear surface morphology evolution driven by stress-assisted surface diffusion. Our results demonstrate that a nominally flat surface profile bounding an elastic stressed solid can rapidly evolve into a crack-like morphology, with smooth tops and sharp, deep grooves. These grooves continue to sharpen and accelerate as they grow. We demonstrate that when the groove depth reaches a critical length, it becomes the mechanical equivalent of an unstable crack. We further show how the stress-driven surface instability maps formally onto classical fracture mechanics. Unlike in classical fracture mechanics, which starts by assuming a crack exists, the present analysis accounts for crack nucleation and propagation based upon a single unified theory.

An outline of our analysis is as follows. A boundary integral equation is developed for a uniaxially stressed, semi-infinite solid bounded by a periodic surface profile. The chemical potential along the surface is defined in terms of the local strain energy density of the surface and the surface energy and is determined based upon the thermodynamics of elastically stressed solids (e.g. Larché and Cahn, 1985). We assume that the dominant matter transport mechanism for surface morphology evolution is surface diffusion. The boundary element procedure is used to solve the elastic equation in conjunction with a finite element procedure for the surface evolution equation. This results in a hybrid method that combines the best features of both procedures. Cubic B-splines are used in both the boundary element and finite element procedures to suppress possible numerical instabilities. A Houbolt integration scheme is used to solve for the temporal evolution of the surface shape.

\section{PROBLEM FORMULATION}

\section{Elastic analysis}

Consider a two-dimensional, semi-infinite solid with a traction-free surface lying along $y=h(x)$, where the solid is in the region $-\infty<y<h(x)$. We assume that the surface profile is periodic with wavelength $\lambda$, i.e. $h(x)=h(x+\lambda)$, and the solid is subjected to a lateral bulk stress $\sigma^{\infty}$ (Fig. 1). We further assume that the wavelength of the lateral bulk stress $\sigma^{\infty}$ is much longer than the wavelength of periodic profile $h(x)$. In a periodic cell, the displacement field can be written as

$$
u_{i}(\mathbf{x})-\varepsilon_{i j}^{\infty} x_{j}+u_{i}^{c}(\mathbf{x})
$$

where $\mathbf{x}$ is an arbitrary point and $i, j=1,2 ; \varepsilon^{\infty}$ is the bulk strain field associated with a uniform bulk stress $\sigma^{\infty}$ that gives rise to a linear displacement field over the periodic cell and

$$
u_{i}^{c}(x, y)=u_{i}^{c}(x+\lambda, y)
$$

is the component of the displacement field that is periodic in the lateral direction. Based on (1), the surface traction field can be similarly written as

$$
t_{i}(\mathbf{x})=\sigma_{i j}^{\infty} n_{j}(\mathbf{x})+t_{i}^{c}(\mathbf{x}),
$$

where $n$, represents the direction cosines of the surface normal and 


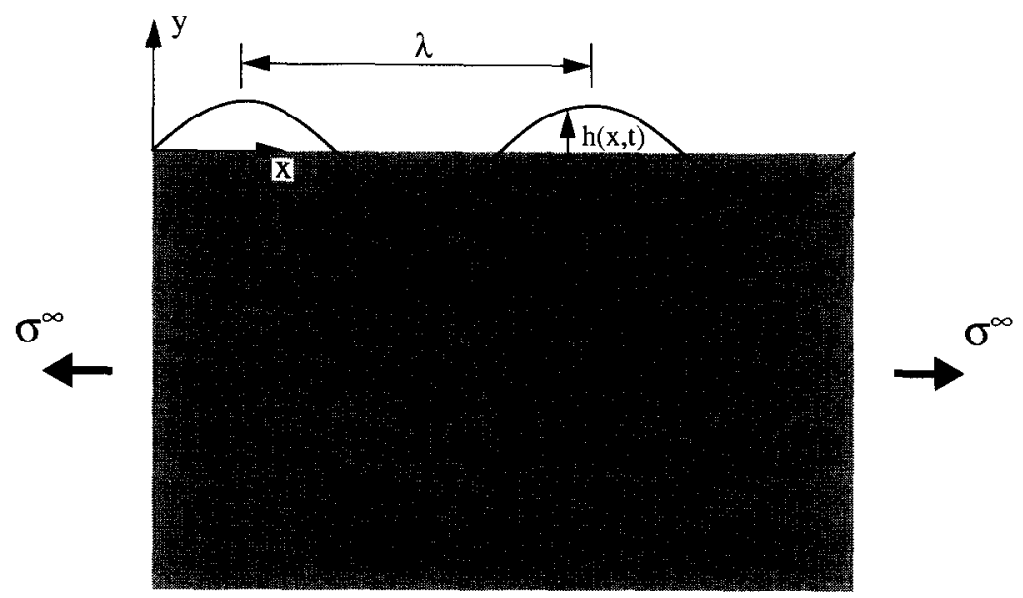

Fig. 1. A two-dimensional semi-infinite solid with a sinusoidal surface profile. The position of the surface $h(x, t)$ is measured with respect to the mean surface height, $y=0$. The wavelength of sinusoidal surface profile is $\lambda$. The solid is subjected to a lateral bulk stress $\sigma^{\infty}$.

$$
\sigma_{i j}^{\infty}=C_{i j k l} \varepsilon_{k l}^{\infty},
$$

in which $C_{i j k l}$ is the isotropic elastic constant tensor.

The integral equation for the displacement $u^{c}$ satisfying the governing equation of elasticity can be expressed as

$$
\begin{aligned}
c_{i j}\left(\mathbf{x}_{0}\right) u_{i}^{c}\left(\mathbf{x}_{0}\right)=\int_{S} \bar{U}_{i j}\left(\mathbf{x}, \mathbf{x}_{0}\right) t_{i}^{c}(\mathbf{x}) \mathrm{d} S(\mathbf{x}) & \\
& -\int_{S} \bar{T}_{i j}\left(\mathbf{x}, \mathbf{x}_{0}\right) u_{i}^{c}(\mathbf{x}) \mathrm{d} S(\mathbf{x})+\hat{U}_{i j} \int_{S} t_{i}^{c}(\mathbf{x}) \mathrm{d} S(\mathbf{x}),
\end{aligned}
$$

where $\mathbf{x}_{0}$ is the source point, $\mathbf{x}$ is the field point and the integrals on $S$ are evaluated over the entire surface. $c_{i j}$ is surface geometry dependent and is simply $c_{i j}=\delta_{i j} / 2$ for points on a smooth surface and $c_{i j}=\delta_{i j}$ for internal points. $\bar{U}_{i j}$ and $\bar{T}_{i j}$ are the elastic kernel functions that satisfy the periodic boundary conditions in the lateral direction. The last term in (5) represents a rigid body motion and $\hat{U}_{i j}$ is a function of materials properties alone.

In order to determine the lateral periodic kernel functions $\bar{U}_{i j}$ and $\bar{T}_{i j}$, we sum the regularly spaced two-dimensional Kelvin solution over an infinite body:

$$
\begin{aligned}
& \bar{U}_{i j}\left(x-x_{0}, y-y_{0}\right)=\sum_{k=\infty}^{\infty} U_{i j}\left(x+k \lambda-x_{0}, y-y_{0}\right) \\
& \bar{T}_{i j}\left(x-x_{0}, y-y_{0}\right)=\sum_{k=-\infty}^{\infty} T_{i j}\left(x+k \lambda-x_{0}, y-y_{0}\right),
\end{aligned}
$$

where $U_{i j}$ and $T_{i j}$ are Kelvin solutions for the displacements and tractions, respectively. By performing the sums associated with the Kelvin solutions (see the Appendix for 
details), we obtain the following explicit expressions for the lateral periodic kernel functions $\bar{U}_{i j}$ and $\bar{T}_{i j}$ for two-dimensional plane strain :

$$
\begin{aligned}
& \bar{U}_{11}=A\left[(3-4 v) \ln (\cosh Y-\cos X)^{1 / 2}+\frac{1}{2} Y \frac{\sinh Y}{\cosh Y-\cos X}\right] \\
& \bar{U}_{12}=-A \frac{1}{2} Y \frac{\sin X}{\cosh Y-\cos X} \\
& \bar{U}_{21}=\bar{U}_{12} \\
& \bar{O}_{22}=A\left[(3-4 v) \ln (\cosh Y-\cos X)^{1 / 2}-\frac{1}{2} Y \frac{\sinh Y}{\cosh Y-\cos X}\right]
\end{aligned}
$$

and

$$
\bar{T}_{i j}=\bar{S}_{j k i} n_{k}
$$

where

$$
\begin{aligned}
& \bar{S}_{111}=B \frac{\pi}{\lambda}\left[(3-4 n) \frac{\sin X}{\cosh Y-\cos X}-Y \frac{\sinh Y \sin X}{(\cosh Y-\cos X)^{2}}\right] \\
& \bar{S}_{222}=B \frac{\pi}{\lambda}\left[2(1-v) \frac{\sinh Y}{\cosh Y-\cos X}+Y \frac{\cosh Y \cos X-1}{(\cosh Y-\cos X)^{2}}\right] \\
& \bar{S}_{221}=B \frac{\pi}{\lambda}\left[-(1-2 v) \frac{\sin X}{\cosh Y-\cos X}+Y \frac{\sinh Y \sin X}{(\cosh Y-\cos X)^{2}}\right] \\
& \bar{S}_{112}=B \frac{\pi}{\lambda}\left[2 v \frac{\sinh Y}{\cosh Y-\cos X}-Y \frac{\cosh Y \cos X-1}{(\cosh Y-\cos X)^{2}}\right] \\
& \bar{S}_{121}=B \frac{\pi}{\lambda}\left[2(1-v) \frac{\sinh Y}{\cosh Y-\cos X}-Y \frac{\cosh Y \cos X-1}{(\cosh Y-\cos X)^{2}}\right] \\
& \bar{S}_{122}=B \frac{\pi}{\lambda}\left[(1-2 v) \frac{\sin X}{\cosh Y-\cos X}+Y \frac{\sinh Y \sin X}{(\cosh Y-\cos X)^{2}}\right] \\
& \bar{S}_{211}=\bar{S}_{121} \\
& \bar{S}_{212}=\bar{S}_{122}
\end{aligned}
$$

and

$$
A=-\frac{1}{8 \pi(1-v) G}, \quad B=-\frac{1}{4 \pi(1-v)},
$$

$X=2 \pi\left(x-x_{0}\right) / \lambda, Y=2 \pi\left(y-y_{0}\right) / \lambda, v$ is the Poisson's ratio and $G$ is the shear modulus of the isotropic solid. The plane strain expressions (7) and (9) can be converted to plane stress by replacing $v$ by $\bar{v}=v /(1+v)$.

Using (7) and (8), we can determine the unknown $u_{i}$ and $t_{i}$ on the surface by solving 
(5). The surface stress is determined by numerical differentiation of the resultant surface displacements. Once the displacement field $u_{i}$ and traction field $t_{i}$ along the surface are known, the internal stresses can also be computed by an additional application of (5) and Hooke's law. The strain energy density on the surface is

$$
\omega={ }_{2}^{1} \varepsilon_{x \beta} \sigma_{\alpha \beta},
$$

where $\alpha, \beta$ are indices that indicate normal and tangent directions.

\section{Surface kinetics}

In order to study the evolution of the surface profile, the chemical potential and material transport kinetics must first be established. The chemical potential $\mu$ along the surface of a stressed solid can be described as

$$
\mu=\mu^{*}+\gamma \kappa \Omega+\omega \Omega+\sigma_{n n} \Omega,
$$

where $\mu^{*}$ is the chemical potential of the flat surface bounding the solid with bulk stress $\sigma^{\infty}, \gamma$ is the isotropic surface energy, $\Omega$ is an atomic volume, $\kappa$ is the surface curvature and $\omega$ is the strain energy density evaluated at the surface. $\sigma_{n n}$ is the stress component normal to the surface. The fourth term in (12) is not present in the context considered here since the surface is assumed to be traction-free. When material transport at the surface is controlled by surface diffusion, the diffusion fluxes are proportional to the surface gradient of the chemical potential (based on the NernstEinstein relation),

$$
J=-\frac{D_{\mathrm{s}}}{\mathrm{k} T} \frac{\partial \mu}{\partial s},
$$

where $D_{s}$ is the surface diffusivity, $\mathrm{k}$ is Boltzmann's constant, $T$ is the absolutc temperature and the derivative with respect to $s$ is evaluated along the surface. The normal velocity of the surface $V_{n}$ is proportional to the divergence of $J$ (Mullins, 1957):

$$
V_{n}=\frac{D_{s} \Omega v_{s}}{\mathrm{k} T} \frac{\partial^{2} \mu}{\partial s^{2}},
$$

where $v_{\mathrm{s}}$ is the number of atoms per unit area of the material in the plane normal to the flux direction.

In the present study, $\sigma_{11}^{\infty}=\sigma^{\infty}$ and $\sigma_{12}^{\infty}=\sigma_{22}^{\infty}=0$, the bulk strain field $\varepsilon_{i j}^{\infty}$ can be written as

$$
\begin{aligned}
& \varepsilon_{11}^{\infty}=\frac{1}{E^{\prime}} \sigma^{\infty} \\
& \varepsilon_{12}^{\infty}=0 \\
& \varepsilon_{22}^{\infty}=-\frac{D^{\prime}}{E^{\prime}} \sigma^{\infty},
\end{aligned}
$$

where $E^{\prime}=E /\left(1-v^{2}\right)$ and $v^{\prime}-v /(1-v)$ for plane strain and $E^{\prime}=E$ and $v^{\prime}=v$ for 
plane stress and where $E$ is Young's modulus. Due to the zero traction boundary condition at the surface, the only stress component along the surface that contributes to the strain energy is $\sigma_{\eta \eta}$ where $\eta$ indicates the direction of the surface tangent. With reference to this local coordinate system, the strain $\varepsilon_{\eta \eta}$ on the surface is

$$
\varepsilon_{\eta \eta}=\frac{\partial u_{\eta}}{\partial \eta}
$$

Applying Hooke's law, the tangential stress $\sigma_{\eta \eta}$ is given by

$$
\sigma_{\eta \eta}=2 E^{\prime} \varepsilon_{\eta \eta}
$$

and the strain energy density along the surface is simply

$$
\omega=\frac{1}{2 E^{\prime}} \sigma_{\eta \eta}^{2}
$$

Since the surface profile is described by $y=h(x)$, we can rewrite (14) in terms of the vertical component of surface profile as

$$
\frac{\partial h}{\partial t}=\Delta \frac{\partial}{\partial x}\left[\left(1+h_{x}^{2}\right)^{-1 / 2} \frac{\partial}{\partial x}(\gamma \kappa+\omega)\right]
$$

where

$$
\begin{gathered}
\Delta=\frac{D_{s} v_{s} \Omega^{2}}{\mathrm{k} T} \\
\kappa=-\frac{h_{x x}}{\left(1+h_{x}^{2}\right)^{3 / 2}}
\end{gathered}
$$

and $h_{x}$ indicates the derivative of the surface profile with respect to $x$. Equation (19) is a nonlinear equation for the evolution of the surface profile.

\section{Numerical implementation}

The evolution of the surface profile is determined by a procedure that combines a numerical solution of the boundary integral equation for elasticity (5) with a finite element technique for solving the nonlinear surface evolution equation (19). We defined a mesh over the free surface of the solid. In order to maintain a high degree of continuity for surface variables, cubic B-spline interpolating functions were employed to describe both the geometric surface and as interpolation functions for the surface displacements $u_{i}$ and tractions $t_{i}$. The cubic B-splines employed in the present study maintain $C^{2}$ continuity between elements of the curve and are nonzero over at most four elements. The Cartesian coordinates of an arbitrary point on surface protile are given by

$$
x=\sum_{i=0}^{N+1} X_{i} B_{i}
$$




$$
y=\sum_{i=0}^{N+1} Y_{i} B_{i}
$$

where $N$ is the number of nodes in the mesh, $X_{i}$ and $Y_{i}$ are the coordinates of the control point at node $i$ and $B_{i}$ are cubic B-spline functions (e.g. de Boor, 1978). The geometric periodic boundary condition is used to determine the positions of the fictitious control points $\left(X_{0}, Y_{0}, X_{N+1}\right.$ and $\left.Y_{N+1}\right)$ in terms of coordinates of interior nodes. Since the B-splines extend over four elements, they give rise to a banded matrix with a bandwidth of seven. Therefore, it is necessary to perform the inversion procedure to find the position of the control points. A similar procedure is applied to represent the surface displacements $u_{i}$ and tractions $t_{i}$.

In the boundary element implementation of (5), both normal Gaussian quadrature with varying numbers of Gauss points and logarithmic Gaussian quadrature are used to evaluated the boundary integrals. Additional details on the solution procedure for the boundary element method, as applied to elasticity, can be found elsewhere (e.g. Brebbia et al., 1984; Henry and Banerjee, 1988).

The Galerkin finite element method is used for transforming the surface kinetics equation from a partial differential equation to an initial value problem. Cubic Bsplines are, again, chosen as the basis functions. The weak formulation of (19) is obtained by multiplying each side of the equation by the set of basis function and integrating over the domain, i.e.

$$
\int_{0}^{\lambda} \frac{\partial h}{\partial t} B_{i} \mathrm{~d} x=\int_{0}^{\lambda} \Delta \frac{\partial R(x, y)}{\partial x} B_{i} \mathrm{~d} x
$$

where

$$
R(x, y)=\left(1+h_{x}^{2}\right)^{-1 / 2} \frac{\partial}{\partial x}(\gamma \kappa+\omega)
$$

Integration by parts eliminates the second derivatives, such that (23) can be expressed as

$$
\int_{0}^{\partial} \frac{\partial h}{\partial t} B_{i} \mathrm{~d} x=-\int_{0}^{\lambda} \Delta R(x, y) \frac{\partial B_{i}}{\partial x} \mathrm{~d} x .
$$

In the numerical implementation of (24), cubic B-splines are also used as interpolation functions for the surface profile $h$, the surface curvature $\kappa$ and the surface strain energy density $\omega$. Four Gauss integration points are used for the evaluation of integrals over each element.

After performing the relevant inversion of the matrices associated with the Bsplines, the resultant kinetics matrix equation can be written schematically as

$$
\frac{\partial \mathbf{h}(t)}{\partial t}=\mathbf{F}(\mathbf{h}, t)
$$

where $\mathbf{F}$ is the force vector for the kinetic equation (19). Equation (25) can be solved by direct integration with either an explicit or implicit time integration method. Based 
on numerical stability and efficiency considerations, the implicit Houbolt integration scheme (e.g. Bathe, 1982) was adopted instead of an explicit method such as the fourth-order Runge-Kutta ( $R-K)$ scheme. The finite difference expansion is

$$
\dot{\mathbf{h}}(t \mid \Delta t)=\frac{1}{6 \Delta t}\left[\begin{array}{lllll}
11 \mathbf{h}(t \mid \Delta t) & 18 \mathbf{h}(t) \mid 9 \mathbf{h}(t-\Delta t) & 2 \mathbf{h}(t-2 \Delta t)
\end{array}\right] .
$$

Substitution of (26) into (25) yields

$$
\mathbf{h}(t+\Delta t)=\frac{1}{11}[6 \Delta t \mathbf{F}(t+\Delta t)+18 \mathbf{h}(t)-9 \mathbf{h}(t-\Delta t)+2 \mathbf{h}(t-2 \Delta t)] .
$$

The numerical stability associated with the integration of the kinetics equation is very sensitive to the choice of the time step. This sensitivity is based upon the presence of high order derivatives of the surface profile $h$ with respect to $x$ in (19). The exact dependence of the sensitivity on the time step for a given choice of the spatial discretization is difficult to assess because (19) does not explicitly show all of the spatial dependencies [i.e. $\omega$ is a complicated, implicit function of $h(x)$ ].

\section{RESULTS AND DISCUSSION}

In order to assess the numerical accuracy and convergence of the boundary integral equation formulation developed in Section 2 , we calculate the strain energy density for a periodic cusped surface similar to those observed in experimental studies of the stress driven surface instability. Chin and Gao (1993) obtained an analytical solution for the strain energy density $\omega$ along a periodic cycloid surface described by

$$
\begin{aligned}
& x=\theta+\beta \sin \theta \\
& y=\beta \cos \theta
\end{aligned}
$$

under a laterial bulk stress $\sigma^{\infty}$, where $0 \leqslant \theta \leqslant 2 \pi$ and $\beta \leqslant 1$. As $\beta$ tends to one, the curvature at the cusp tip diverges. $\omega$ can be written as

$$
\omega(\theta)=\omega_{0} S(\theta),
$$

where $\omega_{0}$ represents the strain energy density when the surface is flat and can be obtained by replacing $\sigma_{t}$ in (18) with $\sigma^{\infty}$, i.c.

$$
\omega_{0}=\frac{1}{2 E^{\prime \prime}}\left(\sigma^{\infty}\right)^{2} .
$$

The term $S(\theta)$ is the geometric factor

$$
S(\theta)=\left(\frac{1-\beta^{2}}{1+2 \beta \cos \theta+\beta^{2}}\right)^{2} .
$$


Table 1. Normalized strain energy density $\mathrm{S}(\pi)$ for the cycloid surface cusp

\begin{tabular}{cccc}
\hline Nodes $N$ & $\beta=0.1$ & $\beta=0.5$ & $\beta=0.9$ \\
\hline 8 & 1.49042 & 8.83427 & 346.01994 \\
16 & 1.49362 & 8.98335 & 358.50773 \\
32 & 1.49382 & 8.99867 & 359.69182 \\
64 & 1.49382 & 8.99991 & 360.69635 \\
128 & 1.49382 & 9.00000 & 360.96064 \\
Analytical (31) & 1.49382 & 9.00000 & 361.00000 \\
\hline
\end{tabular}

The maximum strain energy density occurs at the cusp tip (i.e. $\theta=\pi$ ). Table 1 shows the maximum normalized strain energy density $S(\pi)$ for several values of $\beta$ and several numbers of nodes $N$. The surface nodes are uniformly distributed in $\theta$. Figure 2 shows the relative error as a function of number of nodes. The relative error $\|e\|_{\mathrm{R}}$ is defined as

$$
\|e\|_{\mathbf{R}}=\sqrt{\frac{1}{N} \sum_{i=1}^{N}\left(\frac{\check{\omega}_{i}-\omega_{i}}{\omega_{i}}\right)^{2}}
$$

where $\tilde{\omega}_{i}$ is our calculated strain energy density at node $i$ and and $\omega_{i}$ is the exact strain energy density, determined by Chin and Gao (1993). The results indicate that the relative error $\|e\|_{\mathrm{R}}$ steadily decreases with increasing number of mesh nodes $N$ for all three $\beta$ values. These results verify that the present implementation of the boundary integral equation method is accurate even when the number of nodes is small for the types of problems considered here.

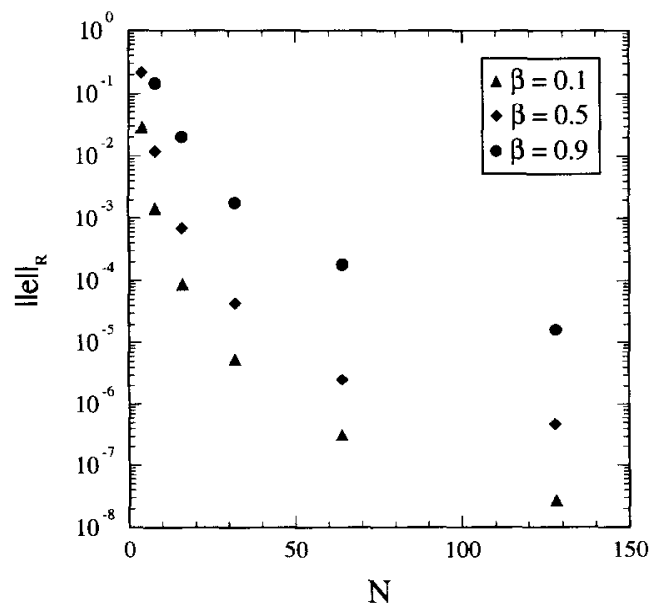

Fig. 2. Convergence rate of uniform mesh refinement on the periodic cycloid surface at $\beta=0.1,0.5$ and 0.9 . The relative crror is in cncrgy normalized by the strain energy density $\omega_{0}$ along the flat surface. 
All of the temporal evolution of the surface profile results are reported in terms of dimensionless stresses and times:

$$
\tau=\frac{\Delta \gamma}{\lambda^{4}} t
$$

and

$$
\Sigma=\sigma^{\infty} \sqrt{\frac{\lambda}{\gamma E}} .
$$

The parameter $\Sigma$ reflects the importance of the elastic strain energy relative to the surface energy.

In order to compare the numerical results of this study with the simple linear stability (perturbation theory) results (e.g. Asaro and Tiller, 1972; Grinfeld, 1986; Srolovitz, 1989), we first review the perturbation analysis as applied to surface diffusion controlled stress driven surface instabilities. Consider an initial sinusoidal perturbation with amplitude $A_{0}$. The perturbation analysis shows that the amplitude of the perturbation will grow as

$$
A(t)=A_{0} \mathrm{e}^{\alpha t},
$$

where $A$ is the amplitude of sinusoidal surface profile and the growth rate $\alpha$ is defined as

$$
\alpha=\Delta\left[\frac{2\left(\sigma^{\infty}\right)^{2} k^{3}}{E^{\prime}}-\gamma k^{4}\right]
$$

and $k(=2 \pi / \lambda)$ is the wave number of the perturbation. The linear stability theory predicts that the surface amplitude will grow if

$$
\sigma^{\infty} \geqslant \sqrt{\frac{\pi \gamma E^{\prime}}{\lambda}} .
$$

Recasting this in terms of the nondimensional stress $\Sigma$, we find that the surface is unstable beyond a critical stress $\Sigma_{c}$

$$
\Sigma \geqslant \Sigma_{\mathrm{c}}=\sqrt{\frac{\pi}{1-v^{2}}}
$$

or $\Sigma_{\mathrm{c}}=1.88$ for $v=1 / 3$.

In this study, the initial surface profile corresponds to a small amplitude sinusoidal surface perturbation $A_{0} / \lambda=0.05$. We also assume that this stress state corresponds to plane strain and Poisson's ratio $v=1 / 3$. Figure 3 shows the temporal evolution of the surface profile of a uniaxially stressed solid with $\Sigma=3$. The surface profile evolves slowly from the initial cosine curve towards one with a pronounced groove at the position of the minimum or valley in the initial surface profile. Once this groove forms, it grows rapidly. Two small bumps form immediately adjacent to the deep groove at long times. 


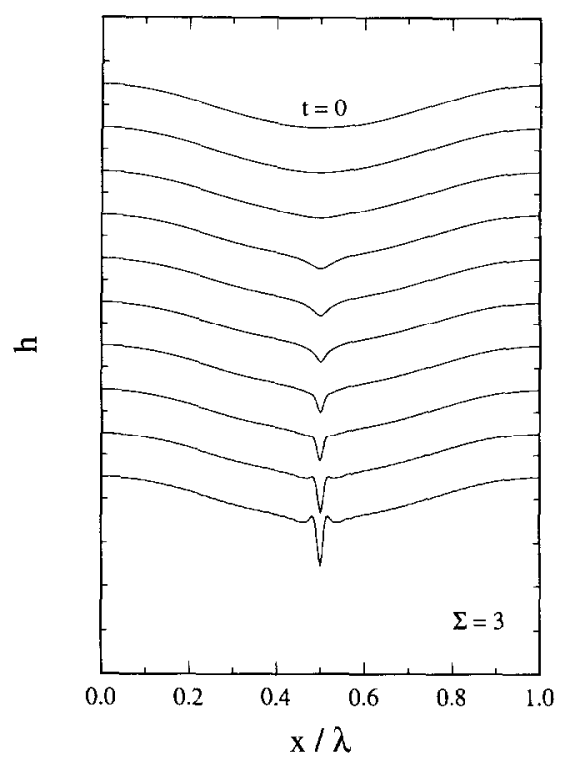

Fig. 3. The temporal evolution of the surface profile under the influence of a bulk stress $\Sigma=3$. The initial surface profile is a cosine wave with an amplitude to wavelength ratio of $A_{0} / \lambda=0.05$. The different curves correspond to $t / \tau=0,0.5 \times 10^{-3}, 1 \times 10^{-3}, 1.5 \times 10^{-3}, 1.55 \times 10^{-3}, 1.56 \times 10^{-3}, 1.565 \times 10^{-3}, 1.567 \times 10^{-3}$, $1.569 \times 10^{-3}$ and $1.571 \times 10^{-3}$ from top to bottom

(The very fine scale oscillations in the two latest time profiles in Fig. 3 indicate the beginning of a numerical instability. This is not a central problem, however, since the dominant sharp, deep groove morphology is evident at times much before this instability develops. This instability can be avoided by combining the B-splines with an adaptive mesh technique.)

The formation of the groove may be attributed to the fact that the strain energy density is highest at the position of the minimum (valley) in the initial surface profile. Since the chemical potential has a term which is linear in the strain energy density (12), matter diffuses away from the minimum and, hence, the groove deepens. As the groove becomes deeper, the stress (and strain energy) concentration at the bottom of the groove increases and the rate of groove growth accelerates. The bumps that form adjacent to the deep groove are regions in which matter diffusing out of the grooves accumulate. Their presence is a common feature of surface diffusion controlled surface morphology evolution (e.g. Mullins, 1957).

The temporal evolution of the surface profile is shown in Fig. 4 for $\Sigma=2.5$. The main features of the evolving groove are similar to those found when $\Sigma=3$ (Fig. 3); however, the surface profile evolves more slowly. The small bumps that form adjacent to the deep groove in the $\Sigma=3$ case are not present when $\Sigma=2.5$. In this case, the transition from the groove to the nearly flat surface (see the latest time curve in Fig. 4 ) is much sharper then seen when $\Sigma=3$. Therefore, the surface tension term $\lambda \kappa$ in the chemical potential (12) is larger at that point for $\Sigma=2.5$ than $\Sigma=3$ and hence matter is transported away from this point at a higher rate. Since matter is transported away from that transition region faster when $\Sigma=2.5$, there is little accumulated 


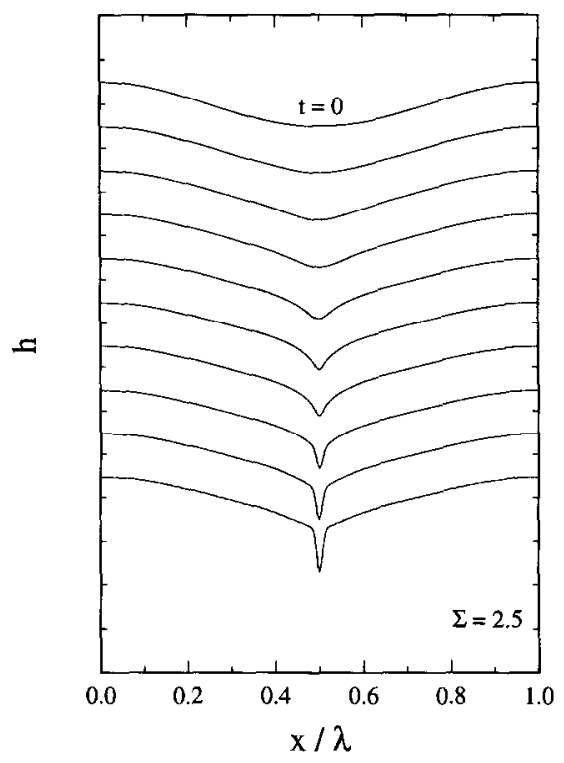

Fig. 4. The temporal evolution of the surface profile under the influence of a bulk stress $\Sigma=2.5$. The initial surface profile is a sine wave with an amplitude-wavelength ratio of $A_{0} / \lambda=0.05$. The different curves correspond to $t / \tau=0,2 \times 10^{-3}, 4 \times 10^{-3}, 5 \times 10^{-3}, 6 \times 10^{-3}, 6.3 \times 10^{-3}, 6.32 \times 10^{-3}, 6.35 \times 10^{-3}, 6.36 \times 10^{-3}$ and $6.37 \times 10^{-3}$ from top to bottom.

matter and hence the bumps seen in the $\Sigma=3$ case do not form. This result demonstrates that when the applied stress is reduced, the smoothing effects of surface tension are more pronounced. This effect also explains why the grooves are wider (i.e. the tip curvature is reduced) when the applied stress is reduced. The effect of stress on the evolution of groove morphology may be seen more clearly in Fig. 5, where we show the groove shape for $\Sigma=3$ and 2.5 on an expanded scale using constant time increments. These results confirm that the groove growth rate accelerates as the groove deepens. As the groove deepens, it also sharpens (i.e. its radius of curvature $\rho=1 / \kappa$ decreases), as shown in Fig. 6 . The radius of curvature $\rho$ decreases gradually until the groove forms and then rapidly approaches zero as the groove sharpens.

The amplitude of the surface profile $\left(h_{\max }-h_{\min }\right)$ is shown as a function of time in Fig. 7 for several values of $\Sigma>\Sigma_{\mathrm{c}}$ [see (33)]. In all cases, the amplitude growth rate rapidly accelerates with time and appears to diverge at a time which is a function of the applied stress. When the profile is smooth and the amplitude is small, the amplitude grows exponentially, as predicted by the linear stability theory. The growth rate accelerates as a well-defined groove forms. The groove sharpens as it continues to grow. Since the groove growth accelerates as the groove grows and sharpens, a positive feedback is established that leads to the divergence of the growth rate at a finite time. The time at which the growth rate diverges decreases with increasing applied stress $\Sigma$.

The results presented in the present study were obtained by fixing $\lambda$ and varying the applied stress. For all stresses above a critical value, the groove growth rate increases with incrcasing stress $\sigma^{\infty}$. This is consistent with the linear stability analysis 


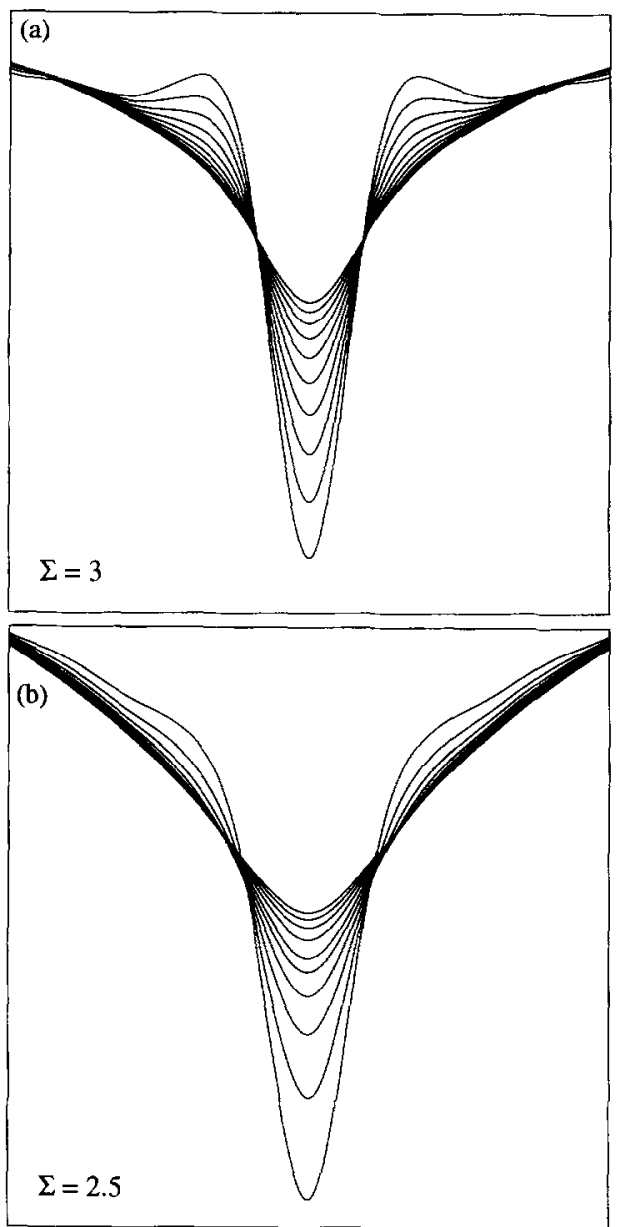

Fig. 5. The groove evolution during the later stage of groove growth. The profiles correspond to a constant time increments for: (a) $\Sigma=3$; and (b) $\Sigma=2.5$.

which shows that the initial growth rate is proportional to the square of the applied stress minus a constant. We note that the growth rate does have a maximum with respect to wave length (at fixed stress) at a finite wavelength even though no such maximum growth rate with respect to applied stress (at fixed wavelength) exists at finite stress.

In order to ensure that the rapid growth rate observed in Fig. 7 is real, a very fine time step $\Delta \tau=10^{-7}$ was employed. Typically there are over 100 time increments in the nearly vertical sections of each of the curves in Fig. 7. Increasing the number of mesh points made no qualitative change in the shape of the curves in Fig. 7. Therefore, the extraordinarily rapid groove growth that occurs is not attributable to the time and mesh discretizations employed.

The present nonlinear analysis clearly shows that the formation of the deep, cracklike groove morphology is a natural consequence of stress driven surface diffusion. 


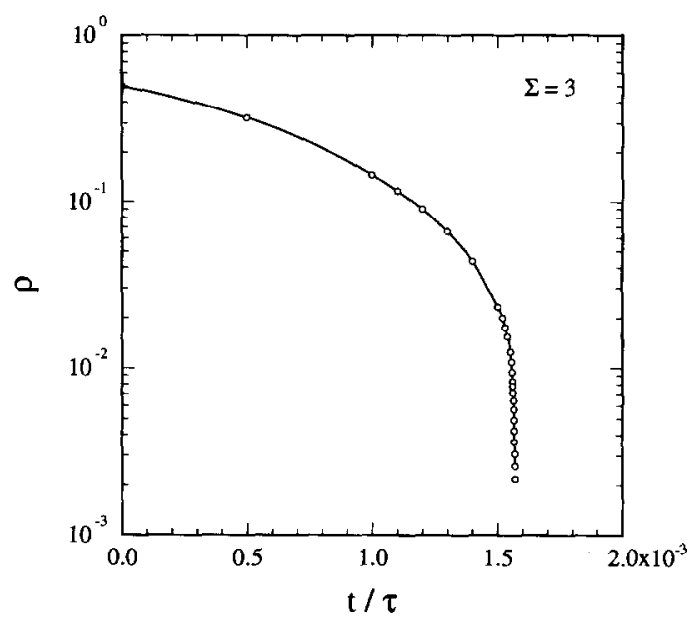

Fig. 6. The temporal evolution of the radius of curvature at the groove tip $\rho$ for the $\Sigma=3$ case.

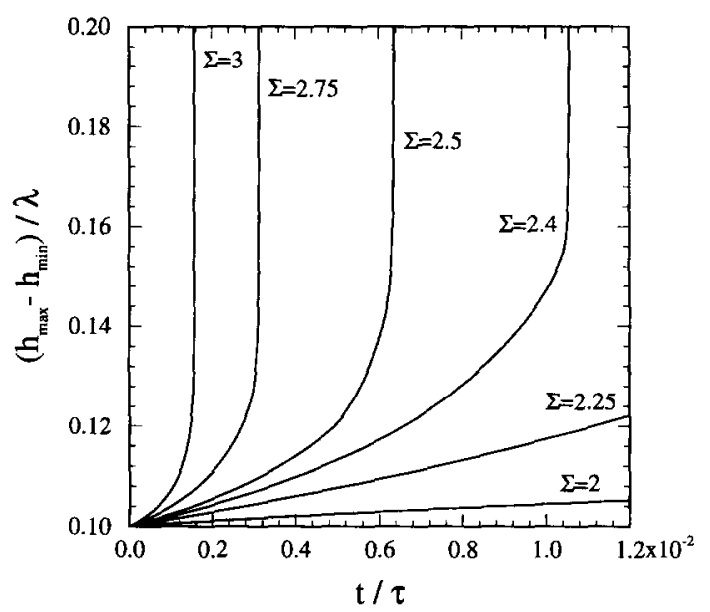

Fig. 7. The time dependence of the amplitude of the surface profile $\left(h_{\max }-h_{\min }\right) / \lambda$ under the influence of applied stress $\Sigma(2.0,2.25,2.4,2.5,2.75$ and 3.0$)$. The amplitude of the initial surface perturbation ratio $A_{0} / \lambda=0.05$. The condition for unstable growth of the surface profile is $\Sigma>1.88$.

The present results also suggest that the groove sharpens as it propagates and accelerates. This acceleration can not go on indefinitely and must be limited by the inherent discreteness of the atomic lattice. An atomically sharp groove is a crack. The cracklike form of the stress-driven surface grooves suggests that there may be a connection between the stress driven surface instability and the fracture process. Before the explosive growth in the groove depth near the threshold, the groove growth can be thought of as either crack nucleation or sub-critical crack growth. Since the growth rate is very high near the threshold time, we use the following procedure to identify the onset of the fracture process: (1) identify the threshold time $\tau^{c}$ where $\mathrm{d}\left(h_{\max }-h_{\min }\right) / \mathrm{d} \tau$ appears to diverge (this is unambiguous, as seen in Fig. 7); and (2) identify the critical 


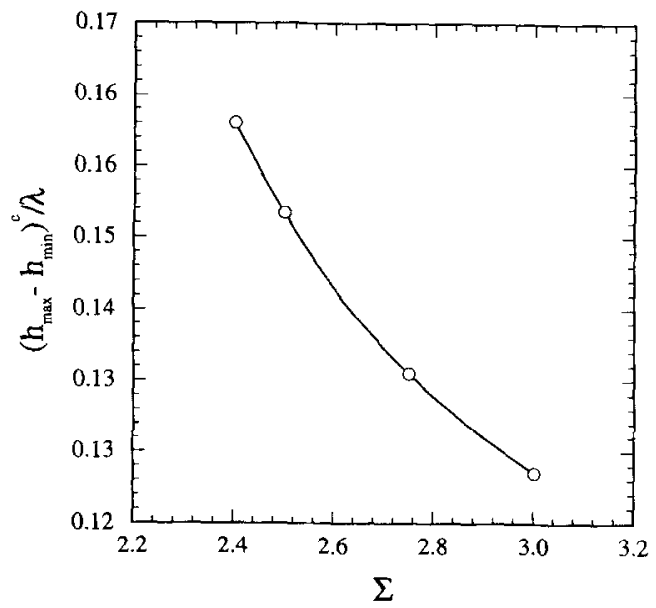

Fig. 8. The dependence of the critical profile amplitude $\left(h_{\max }-h_{\operatorname{mn}}\right)^{c} / \lambda$ on the applied stress $\Sigma$.

groove length as $h_{\max }-h_{\min }$ at $\tau=0.995 \tau^{c}$. Figure 8 shows this dependence of the critical $\left(h_{\max }-h_{\min }\right)$ on $\Sigma$. It is seen that the $\left(h_{\max }-h_{\min }\right)^{c}$ steadily decreases with increasing $\Sigma$. The critical groove length $\left(h_{\max }-h_{\min }\right)^{c}$ depends on the choice of the deviation from $\tau_{c}$ where $\left(h_{\max }-h_{\min }\right)^{c}$ is measured; however, we note that small variations primarily shift the curve in Fig. 8 to higher or lower $h_{\max }-h_{\min }$ without substantially changing its shape.

$\left(h_{\max }-h_{\min }\right)^{c}$ must be related to the critical crack length $a^{*}$ associated with fracture process. Since the $h_{\max }-h_{\min }$ is not the crack length $a$ in an ideal crack configuration, there are several geometric effects that must be accounted for in order to determine the effective crack length $a$ based on $h_{\max }-h_{\min }$ (see Fig. 9). As described above, the initial surface profile is sinusoidal with amplitude $A_{0}=0.05 \lambda$. Furthermore, in the time scale of the simulations, very little change in the surface profile occurs except near the groove. The presence of this finite initial amplitude profile must be accounted for in converting between $\left(h_{\max }-h_{\min }\right)^{c}$ and $a^{*}$. Figure 5 shows that the shape of the crack is dependent on the applied loading and hence this shape effect must also be accounted for in terms of an effective crack length. Therefore, instead of simply subtracting the initial profile amplitude from $h_{\max }-h_{\min }$ to determine the effective crack length $a$, a fitting procedure based upon fracture mechanics is employed.

The periodic sharp groove type geometry found in the present analysis may be

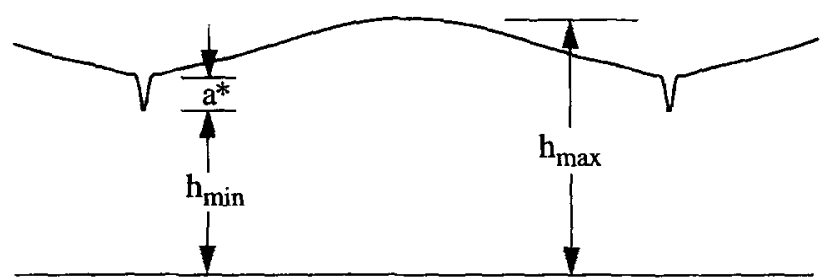

Fig. 9. The surface morphology after the formation of the deep, crack-like groove. $a^{*}$ is the effective groove depth and is small compared to periodic wavelength $\lambda$. 
analyzed in terms of a periodic array of parallel edge cracks. The stress intensity factor $K_{\mathrm{I}}$ for parallel edge cracks in a half-plane with depth $c$ and spacing $2 b$, loaded in tension by a unform normal stress $\sigma^{\infty}$ at infinity (e.g. Benthem and Koiter, 1973), is

$$
K_{\mathrm{I}}=B \sigma^{\infty} \sqrt{\frac{\pi b c}{s}}
$$

where $s=b+c$ and $B$ is a function that depends on the crack length and crack spacing. For $c / s \ll 1, B$ can be expressed as

$$
B \approx 1.122\left[1+\frac{1}{2} \frac{c}{s}-1.694 \frac{c^{2}}{s^{2}}-4.860 \frac{c^{3}}{s^{3}}+O\left(\frac{c^{4}}{s^{4}}\right)\right] .
$$

For the results presented above, the groove depth is always small relative to the periodic length $\lambda$ and hence the expansion in (40) is valid. Therefore, the stress intensity factor for the crack-like groove shown in Fig. 8 can be approximately represented as

$$
K_{\mathrm{I}}=\chi \sqrt{\pi a} \sigma^{\infty},
$$

where $\chi=1.122$ and $a$ is the effective crack length. We note that although the periodic array of grooves examined in the present study are not exactly equivalent to the periodic array of cracks assumed in deriving (40) and (41), the deviation in the stress intensity factors are small. This is because the main differences in the profiles are far from the crack tip, where the strain energy density is very low.

The strain energy release rate for plane strain is

$$
G=\frac{\left(1-v^{2}\right) K_{1}^{2}}{E} .
$$

In an elastic (i.e. nondissipative) material, crack growth occurs when the Griffith condition is satisfied:

$$
G \geqslant 2 \gamma
$$

where, as before, $\gamma$ is the surface energy of the solid. In other words, crack growth occurs when the crack length exceeds a stress-dependent critical size $a^{*}$

$$
a \geqslant a^{*}=\frac{2 \gamma E}{\pi \chi^{2}\left(1-v^{2}\right)\left(\sigma^{\infty}\right)^{2}} .
$$

Rewriting this in terms of the dimensionless variables introduced in (34), we find

$$
\frac{a^{*}}{\lambda}=A_{1}^{*} \frac{1}{\Sigma^{2}},
$$

where 


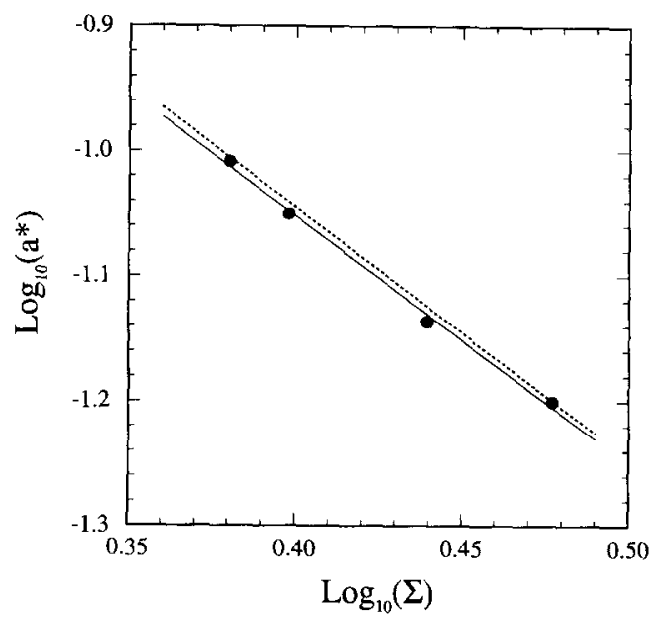

Fig. 10. The dependence of the effective groove depth $a^{*}$, defined in (48), on the applied stress $\Sigma$. The solid curve represents the best fit line (from the numerical data points), while the dotted curve represents the reference state for an edge crack, as defined in (37).

$$
A_{\mathrm{l}}^{*}=\frac{2}{\pi \chi^{2}\left(1-v^{2}\right)},
$$

when $v=1 / 3, A_{1}^{*}=0.569$.

Based on (45), the results in Fig. 8 were fit using the following relation in order to determine the effective groove depth $a^{*}$ :

$$
\frac{\left(h_{\max }-h_{\min }\right)^{c}}{\lambda}=A_{1} \frac{1}{\Sigma^{2}}+\frac{A_{2}}{\lambda}
$$

where the additional term, $A_{2} / \lambda$, was added to account for the finite amplitude of the initial sinusoidal surface profile and crack shape effects. The best fit values of $A_{1}$ and $A_{2}$ were found to be $A_{1}=0.560$ and $A_{2} / \lambda=0.062$. This value of $A_{1}$ agrees with the value of $A_{1}^{*}$ [determined from the stress intensity factor of a crack, (46)] to an accuracy of $1.6 \% . A_{2} / \lambda$ found in the fitting procedure is in reasonable agreement with the initial surface profile amplitude $A_{0} / \lambda=0.05$. The $20 \%$ discrepancy is attributable to other geometrical factors (as described above) and to our choice of the value of $\tau$ at which to define the critical crack length, $\left(h_{\max }-h_{\min }\right)^{c}$. Thus the effective nondimensional critical groove depth $a^{*}$ is defined [combine (45) and (47)] as

$$
a^{*}=\left(h_{\max }-h_{\min }\right)^{c}-A_{2} \text {. }
$$

Figure 10 shows the dependence of the critical crack length $a^{*}$ on the stress $\Sigma$. Here the individual data points represent the numerical results shown in Fig. 8 and using (48), while the solid line represents the best linear fit to these data points or, equivalently, the best fit to the functional form $a^{*} / \lambda=q \Sigma^{p}$. The dotted line represents the result from the fracture mechanics calculation, (45). A comparison of the results of the fit to the fracture mechanics prediction is shown in Table 2.

The excellent agreement between our numerical results and the predictions of 
Table 2

\begin{tabular}{ccc}
\hline & Present analysis & Fracture mechanics \\
\hline$p$ & -1.979 & -2.000 \\
$q$ & 0.548 & 0.569 \\
\hline
\end{tabular}

fracture mechanics clearly demonstrates that the condition for explosive groove growth is that the combination of applied stress and crack length must satisfy the classical Griffith criterion for fracture. This agreement is obtained with no adjustable parameters. Once the groove length meets the classical fracture criterion, it will continue to propagate by bond breaking instead of by surface diffusion. This is the normal unstable crack growth of elastic fracture mechanics. $\Lambda$ t this point, crack growth rates are limited by the speed of sound. The groove growth rate diverges when its length reaches the critical length $a^{*}$. Although this result was obtained based upon surface diffusion kinetics instead of the normal bond breaking fracture process, the Griffith condition is satisfied. This demonstrates that the fracture criterion is essentially thermodynamically controlled rather than kinetically controlled.

In order to further explore the relationship between the growing grooves and cracks, we analyse the stress field ahead of the groove and compare with that for a sharp crack. For a sharp crack under a remote load, the stress distribution ahead of the crack tip is

$$
\sigma_{i j}=\frac{K_{\mathrm{I}}}{(2 \pi r)^{1 / 2}} f_{i j}(\theta),
$$

where $r$ is the distance from the crack tip and $f_{i j}(\theta)$ describes the angular dependence of the stress field. While the $r^{-1 / 2}$ dependence of the stress field is characteristic of a crack-like singularity, if the crack is not perfectly sharp this power-law dependence is only valid in the far field. On the other hand, the stress field ahead of a "V" shaped notch has a singularity of the form:

$$
\sigma_{x x} \propto \frac{1}{r^{x}}
$$

where $\alpha \leqslant 0.5$ depends on the angle at the root of the notch. We use the exponent $\alpha$ to provide a measure of how crack-like a groove is as it goes from being relatively broad $(\alpha \rightarrow 0)$ to perfectly sharp $(\alpha \rightarrow 1 / 2)$.

Figure 11 shows the stress distribution ahead of the groove tip as a function of $r / \lambda$ at three different times $\left(\tau=1.0 \times 10^{-3}, 1.5 \times 10^{-3}\right.$ and $\left.1.565 \times 10^{-3}\right)$. When $\tau=1.0 \times 10^{-3}$, the stress ahead of the valley or groove is slightly elevated (concentrated) but does not vary with distance over two decades in $r / \lambda$ and then decays to the bulk, applied stress. The stress concentration increases with time and groove length. When the groove approaches its threshold length or time ( $\left.\tau=1.565 \times 10^{-3} \approx \tau^{*}\right)$, a crack-like groove starts to grow and a linear relation between $\log (\Sigma)$ and $\log (r)$ is observed. Again, at large $r$, the curve approaches the applied stress $\Sigma^{\infty}$. 


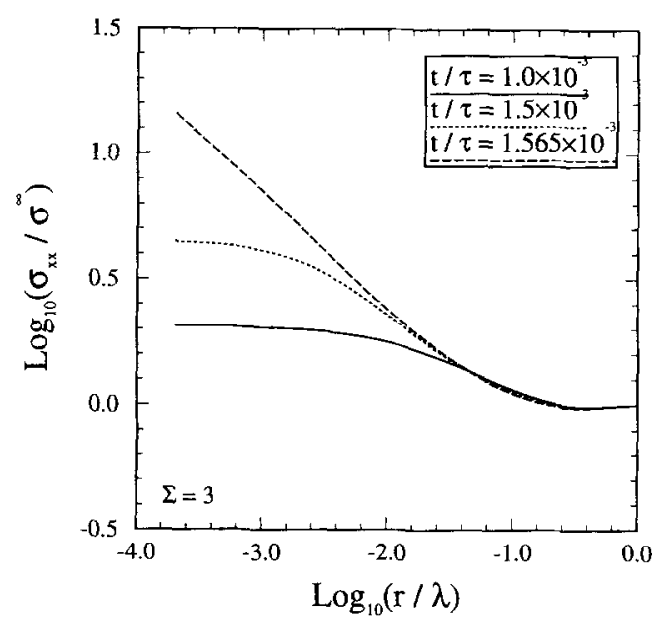

Fig. 11. The stresses distribution as a function of $r / \lambda$ at three different stages of growth $\left(t / \tau=1.0 \times 10^{-3}\right.$. $1.5 \times 10^{-3}$ and $1.565 \times 10^{-3}$ ) for $\Sigma=3 . r / \lambda$ is the distance ahead of the groove tip where the stress is measured.

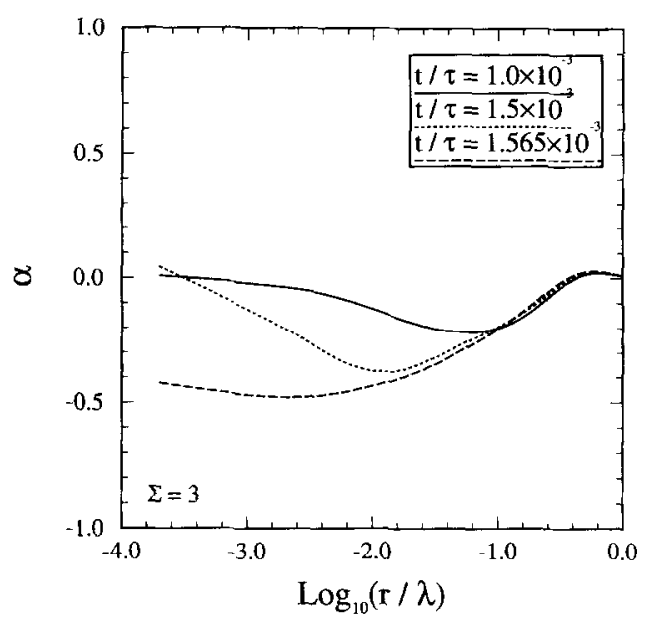

Fig. 12. The variation of the stress singularity $\alpha$ as a function of $r / \lambda$ at three different stages of growth $\left(t / \tau=1.0 \times 10^{-3}, 1.5 \times 10^{-3}\right.$ and $\left.1.565 \times 10^{-3}\right) ; \Sigma=3$.

In order to determine the $\alpha$ variation ahead of the groove tip, we calculate the slope of the three curves in Fig. 11. The dependence of the stress singularity $\alpha$ (or slope of Fig. 11) as a function of $r / \lambda$ is shown in Fig. 12. The stress singularity $\alpha$ is not a constant, i.e. it varies with $r$. Therefore we have adopted three independent procedures to obtain the appropriate value of $\alpha$ : (1) use the minimum value of $\alpha$; (2) use the value of $\alpha$ one groove radius of curvature ahead of the groove; and (3) use the value of $\alpha$ at a fixed distance ahead of the groove tip $r / \lambda=0.004$. All three values of $\alpha$ are plotted in Fig. 13 as a function of time. In all three cases, $\alpha$ starts off at a small value, slowly rises and tends to 0.5 at a fixed time. $\alpha$ goes to 0.5 at the same time that the 


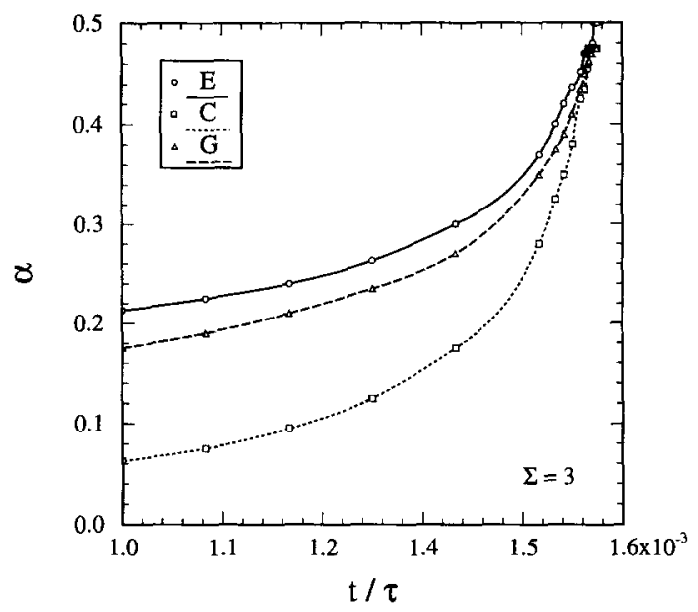

Fig. 13. The time dependence of stress singularity $\alpha$ as a function of $t / \tau$. Each curve corresponds to different method to determine $\alpha$ : E- at the position where $\alpha$ is a minimum; C-at $r$ equals the radius of curvature at the groove tip; $\mathrm{G}-$-at a fixed distance $r / \lambda=0.004$.

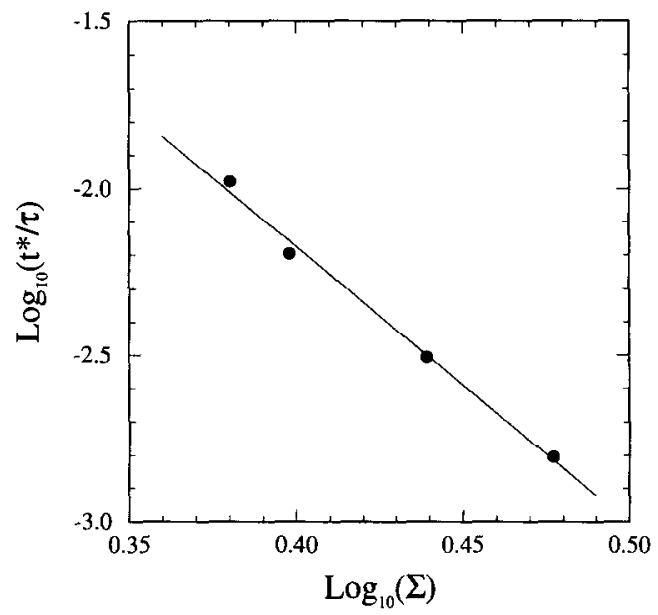

Fig. 14. The failure time $t^{*} / \tau$ as function of applied stress $\Sigma$. The solid curve represents the best linear fit to the numerical data $(\bullet)$, as defined in (51).

groove approaches its critical length $a^{*}$. Therefore, we conclude that the groove does not behave like a crack until it reaches its critical length.

The relation between failure time and the applied stress $\Sigma$ is shown in Fig. 14 where the numerical data are obtained from Fig. 10. If we fit these data to a power law of the form

$$
\tau^{*}=\Sigma^{-n}
$$

the best fit corresponds to $n=8.3$. An inferior fit is obtained using an exponential relationship betwecn failure time and applied stress. This curve shows that the time 
necessary for crack nucleation and growth to instability increases quickly with the applied stress.

\section{SUMMARY}

We have performed a nonlinear analysis of the temporal evolution of the surface morphology of a stressed solid based on a general parametric description of the surface shape. The results demonstrate that the surfaces of elastic solids are unstable against the nucleation and growth of cracks, even in an initially defect-free solid with a surface which is nearly flat. The cracks nucleate as a result of a very general surface instability. The rate at which this surface instability occurs depends on the material transport kinetic mechanism that is operative. The surface instability creates a groove that sharpens as it grows deeper. As it sharpens and deepens, the groove growth rate accelerates until the groove reaches a specific length (or time) where the growth rate diverges. Comparison of these results with predictions of linear elastic fracture mechanics shows that the critical length is in excellent agreement with the classical Griffith fracture criterion, with no adjustable parameters. The stress field ahead of the growing groove becomes increasingly singular as the groove grows. Once the critical groove length is achieved, the stress field ahead of the groove approaches the inverse square root dependence on distance from the tip which is characteristic of a sharp crack. Therefore, the sub-critical groove is not simply a short crack.

\section{ACKNOWLEDGEMENTS}

The authors gratefully acknowledge helpful discussions with L. B. Freund and W. L. Winterbottom. DJS gratefully acknowledges the hospitality of The Weizmann Institute of Science and the support of the Michael Visiting Professorship. This research was supported by the Air Force Office of Scientific Research, Grant No. AFOSR-90-0141.

\section{REFERENCES}

Asaıo, R. J. and Tiller, W. A. (1972) Interface morphology development during stress corrosion cracking: part 1. Via surface diffusion. Metall. Trans. 3, 1789-1796.

Bathe, K. J. (1982) Finite Element Procedures in Engineering Analysis. Prentice-Hall, Englewood Cliffs, NJ.

Benthem, J. P. and Koiter, W. T. (1973) Asymptotic approximations to crack problems. Methods of Analysis and Solutions of Crack Problems, pp. 131-178. Noordhoff, Leyden.

Berrehar, J., Caroli, C., Lapersonne-Meyer, C. and Schott, M. (1992) Surface patterns on single-crystal films under uniaxial stress : experimental evidence for the Grinfeld instability. Phys. Rev. B 46, 13487-13495.

de Boor, C. (1978) A Practical Guide to Splines. Springer, New York.

Brebbia, C. A., Telles, J. C. F. and Wrobel, L. C. (1984) Boundary Element Techniques. Springer, Berlin.

Chin, C.-H. and Gao, H. (1993) Stress singularities along a cycloid rough surface. Int. J. Solids Struct. 30, 29833012. 
Chuang, T.-J. and Fuller, E. R. (1992) Extended Charles-Hillig theory for stress-corrosion cracking of glass. J. Am. Ceram. Soc. 75, 540-545.

Freund, L. B. and Jonsdottir, F. (1993) Instability of a biaxially stressed thin film on a substrate due to material diffusion over its free surface. J. Mech. Phys. Solids 41, 1245-1264.

Gao, H. (1991) A boundary perturbation analysis for elastic inclusions and interfaces. Int. $J$. Solids Struct. 28, 703-725.

Grilhe, J. (1993) Study of roughness formation induced by homogeneous stress at the free surface of solids. Acta Metall. 41, 909-913.

Grinfeld, M. A. (1986) Instability of the separation boundary between a non-hydrostatically stressed elastic body and a melt. Sov. Phys. Dokl. 31, 831-834.

Henry, D. P. and Banerjee, P. K. (1988) A new boundary element formulation for twodimensional and 3-dimensional thermoelasticity using particular integrals. Int. J. Numer. Meth. Engng B 26, 2061-2077.

Heidug, W. K. (1991) A thermodynamic analysis of the conditions of equilibrium at nonhydrostatically stressed and curved solid-fluid interfaces. J. Geophys. Res. 96(21), 909-921.

Hillig, W. B. and Charles, R. J. (1965) Surfaces, stress-dependent surface reactions, and strength. High-Strength Materials, pp. 682-705. Wiley, New York.

Hirth, J. P. and Lothe, J. (1982) Theory of Dislocations. Wiley, New York.

Korsukov, V. E., Luk'yanenko, A. S., Obidov, B. A. and Svetlov, V. N. (1992) Transformation of the surface of the amorphous alloy $\mathrm{Fe}_{70} \mathrm{Cr}_{15} \mathrm{~B}_{15}$ under stress. JETP Lett. 55, 621-623.

Larché, F. C. and Cahn, J. W. (1985) The interactions of composition and stress in crystalline solids. Acta Metall. 33, 331-357.

Mullins, W. W. (1957) Theory of thermal grooving. J. Appl. Phys. 28, 333-339.

Mullins, W. W. (1959) Flattening of a nearly plane solid surface due to capillarity. J. Appl. Phys. 30, 77-83.

Ricc, J. R. and Chuang, T.-J. (1981) Energy variations in diffusive cavity growth. $J$. Am. Ceram. Soc. 64, 46-53.

Sih, G. C. (1991) Mechanics of Fracture Iniation and Propagation. Kluwer, Utrecht.

Spencer, R. J., Davis, S. H. and Voorhees, P. W. (1991) Morphological instability in epitaxially strained dislocation-free solid films-nonlinear evolution. Phys. Rev. B 47, 9760-9777.

Spencer, B. J. and Meiron, D. I. (1993). Acta Metall. (in press).

Spencer, B. J., Voorhees, P. W. and Davis, S. H. (1991) Morphological instability in epitaxially strained dislocation free solid films. Phys. Rev. Lett. 67, 3696-3699.

Srolovitz, D. J. (1989) On the stability of surfaces of stressed solids. Acta Metall. 37, 621-625.

Torii, R. H. and Balibar, S. (1992) Helium crystals under stress: the Grinfeld instability. $J$. Low Temp. Phys. 89, 391-400.

Volkert, C. A. (1993) Private communications.

Yang, W. H. and Srolovitz, D. J. (1993) Crack-like surface instabilities in stressed solids. Phys. Rev. Lett. 71, 1593-1596.

\section{APPENDIX}

Under two-dimensional plane strain conditions, the Kelvin solution for the displacement and stress in an infinite elastic medium are expressed as

$$
\begin{gathered}
U_{i j}\left(\mathbf{x}_{0}, \mathbf{x}\right)=\frac{-1}{8 \pi(1-v) G}\left\{(3-4 v) \ln (r) \delta_{i j}-r_{, i} r_{, i}\right\} \\
S_{j k i}\left(\mathbf{x}_{0}, \mathbf{x}\right)=\frac{-1}{4 \pi(1-v) r}\left\{(1-2 v)\left(r_{, k} \delta_{i j}+r_{, j} \delta_{k i}-r_{, i} \delta_{j k}\right)+2 r_{, i} r_{, j} r_{, k}\right\},
\end{gathered}
$$

where $i, j, k=1,2$ and $r=r\left(\mathbf{x}_{0}, \mathbf{x}\right)$ represents the distance between the load point $\mathbf{x}_{0}$ and the field point $\mathbf{x}$ and its derivatives are taken with reference to the coordinates of $\mathbf{x}$. By performing the sums in (6), we can determine the lateral periodic Green's functions $\bar{V}_{i j}$ and $\bar{S}_{i j k}$. The sums 
associated with the Kelvin solutions of displacement and stress can be decomposed into the following basic kernel function for the two-dimensional case.

The following analytical sums are used to evaluate the summations implicit in (6)-(9) (Hirth and Lothe, 1982):

$$
\begin{gathered}
\sum_{n=-\infty}^{\infty} \frac{n+p}{q^{2}+(n+p)^{2}}=\frac{\pi \sin (2 \pi p)}{\cosh (2 \pi q)-\cos (2 \pi p)} \\
\sum_{n=-\infty}^{\infty} \frac{1}{q^{2}+(n+p)^{2}}=\frac{\pi}{q} \frac{\sinh (2 \pi q)}{\cosh (2 \pi q)-\cos (2 \pi p)} \\
\sum_{n=-\infty}^{\infty} \frac{q^{2}-(n+p)^{2}}{\left[q^{2}+(n+p)^{2}\right]^{2}}=2 \pi^{2} \frac{\cosh (2 \pi q) \cos (2 \pi p)-1}{[\cosh (2 \pi q)-\cos (2 \pi p)]^{2}} \\
\sum_{n=-\infty}^{\infty} \frac{(n+p)}{\left[q^{2}+(n+p)^{2}\right]^{2}}=\frac{\pi^{2}}{q} \frac{\sinh (2 \pi q) \sin (2 \pi p)}{[\cosh (2 \pi q)-\cos (2 \pi p)]^{2}} \\
\sum_{n=-\infty}^{\infty} \ln \left[q^{2}+(n+p)^{2}\right]=\ln [\cosh (2 \pi q)-\cos (2 \pi p)] .
\end{gathered}
$$

